\title{
THE SPHERICAL SAMPLE METHOD IN NEUTRON DIFFRACTION TEXTURE DETERMINATION
}

\author{
J. TOBISCH and H. J. BUNGE \\ Technische Universität Dresden, Sektion Physik, and Zentralinstitut für Festkörperphysik und \\ Werkstofforschung Dresden, der Deutschen Akademie der Wissenschaften zu Berlin, GDR.
}

(Received May 2, 1972, in final form June 28, 1972)

\begin{abstract}
Neutron diffraction proves advantageous as compared to X-ray diffraction in texture analysis because of the lower absorption coefficient for a broad variety of materials especially metals. The spherical sample method is recommended because it yields the most reliable results and it does not require great preparational efforts. The fundamental difference between the spherical sample method in X-ray and in neutron diffraction is discussed.
\end{abstract}

\section{X-RAY AND NEUTRON DIFFRACTION}

Texture measurements usually are carried out by the X-ray diffraction technique. In recent years, however, neutron diffraction became accessible more easily and was applied to texture investigations at an increasing rate ${ }^{1-14}$. Both these methods are very similar to one another. There are, however, some quantitative differences which, in many cases, make neutron diffraction by far superior to X-ray diffraction. A comparison of the quantitative aspects of the two methods was given, for example, in several recent publications. ${ }^{9,15,16}$

Most of the superiorities of neutron diffraction in texture analysis are based on the much lower absorption coefficient for neutrons in a wide variety of materials especially metals. Hence the sample may be taken several millimeters or even centimeters thick without the necessary absorption correction being unduly large. This allows specimens of industrially interested dimensions to be investigated as a whole. Because of the usual inhomogeneity of the textures, such overall sampling is necessary for the calculation of average orientation properties comparable with the macroscopic properties of the material.

Secondly, the relative deviations of the sample from its ideally proposed shape can be kept much smaller in the millimeter range than in the range of a few hundredths or tenths of a millimeter. Hence the remaining absorption correction can be calculated much more precisely than in the X-ray case. Thus it is possible to reduce the experimental errors of texture measurements by almost an order of magnitude without increasing the efforts required in specimen preparation. It is this feature which makes neutron diffraction exceptionally valuable if texture data are to be processed mathematically. ${ }^{17}$

There is, however, one source of error which is not reduced or which may even be increased by neutron diffraction, if the usual transmission and back reflection techniques are employed. In these techniques different areas of the sample are being irradiated at different angular positions, because the sheet plane cuts the primary beam at different angles. Hence at different angular positions of the sample, different lots of crystallites are involved which may exhibit different orientation distributions among themselves, if the texture is not homogeneous. This means that different parts of a pole figure may correspond, strictly speaking, to different textures. This effect may easily be estimated with materials of cubic crystal symmetry. In this case certain integrals taken over the whole pole figure were to vanish if the pole figure was correct. ${ }^{17}$ The deviation from zero provides then a good measure of the inconsistency of the pole figure values among themselves. The inconsistency must be well distinguished from the uncertainty of a pole figure being brought about by the uncertain definition of an inhomogeneous texture, although both are of the same order of magnitude. When only pole figures are examined this difference does not matter. If, however, three-dimensional orientation distribution functions are to be calculated from the pole figure data either by an interational method or 
by solving a system of linear equations for the coefficients, then the inconsistency may give rise to an additional, even enhanced, error. An inhomogeneity effect of this kind frequently occurs in the transverse direction, if metal sheet samples are prepared on a laboratory scale (rolled in strips of $10 \mathrm{~cm}$ or less in width). Since with the usual backreflection and transmission technique of neutron diffraction the optimum sample diameter is about $5-10 \mathrm{~cm}$, considerable parts of the inhomogeneous side regions of the strip may be included.

\section{THE SPHERICAL SAMPLE METHOD}

An inhomogeneity effect of this kind may easily be avoided if the sample is smaller than the cross section of the incident beam. In this case, the same lot of crystallites is being irradiated at any angular position. Furthermore, if the shape of the sample is approximately that of a sphere, then correction for absorption can be neglected, because of the low absorption coefficient. The resulting intensity values are immediately proportional to pole density values, and they are no longer inconsistent with each other arising from inhomogeneity effects. Figure 1 shows a

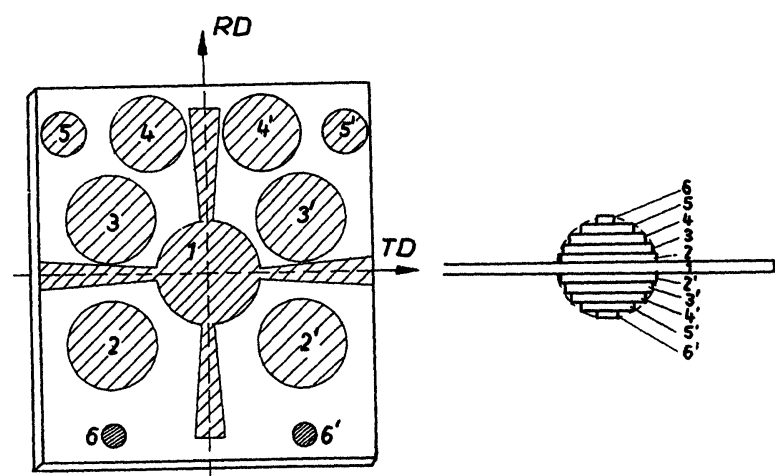

FIGURE 1 Preparation of a "spherical sample" for neutron diffraction texture analysis.

very simple method for constructing a "sphere" out of a sheet material. A "sphere" of this quality is completely sufficient for the spherical sample method in the case of neutron diffraction. The cross-shaped bars of the section number 1 are used to fasten the spherical sample to the goniometer in the same way as sheet samples are fastened. They may give rise to a small residual inhomogeneity effect, but they may also be replaced by some other material soldered or glued to the sample. The scheme of measurement is the same as it is with the sheet specimen, for both the "transmission" and the "back-reflection" ranges. As far as the sample itself is concerned, there is no longer any difference between these two ranges. The division of the whole pole figure into these two ranges is necessary only because of the shadow cast by certain parts of the goniometer.

It is to be emphasized that there is a fundamental difference between the spherical sample method in neutron diffraction and the same method in X-ray diffraction. ${ }^{18,19}$ In the X-ray method, diffraction takes place solely in a small surface layer of the sphere. Therefore, every angular position is being measured with a completely different lot of crystallites. Hence the X-ray method is extremely sensitive to inhomogeneities of the texture. Furthermore, a smooth and well-defined spherical surface is needed. In the neutron method, on the other hand, diffraction takes place in the whole volume of the specimen. Hence inhomogeneity effects are completely eliminated, and there is no need for a good surface.

An appraisal of the intensity of the reflected beam, the absorption and other geometrical conditions show that reasonable diameters for a spherical sample range from about $0.5 \mathrm{~cm}$ to 3 or $4 \mathrm{~cm}$. Furthermore it turned out that a certain amount of material made into a sphere yields a higher pulse rate of the reflected beam than the same volume investigated in the form of a sheet. This is due to the specific aperture conditions in the back-reflection and transmission method. ${ }^{20}$ Thus the spherical sample method, to a certain degree, may reduce the limitations of the neutron diffraction method if only small amounts of material are available.

The pole figures of a textured specimen may be regarded as different "projections" of the threedimensional texture function. Hence there exist certain relationships among them which must be fulfilled exactly if the pole figures were devoid of experimental error. In any real case, however, there will be finite deviations which can be used to define certain error coefficients describing the degree of misfit of the pole figures among one another. ${ }^{17}$ In Figure 2 these error coefficients are shown for an inhomogeneous brass-specimen measured by the usual back-reflection and transmission method (curve a). It has been suggested that the higher order coefficients corresponded to statistical errors, whereas the strong increase towards lower orders was due to systematical errors. $^{21}$ The latter could have been brought about by the texture inhomogeneities described above. 


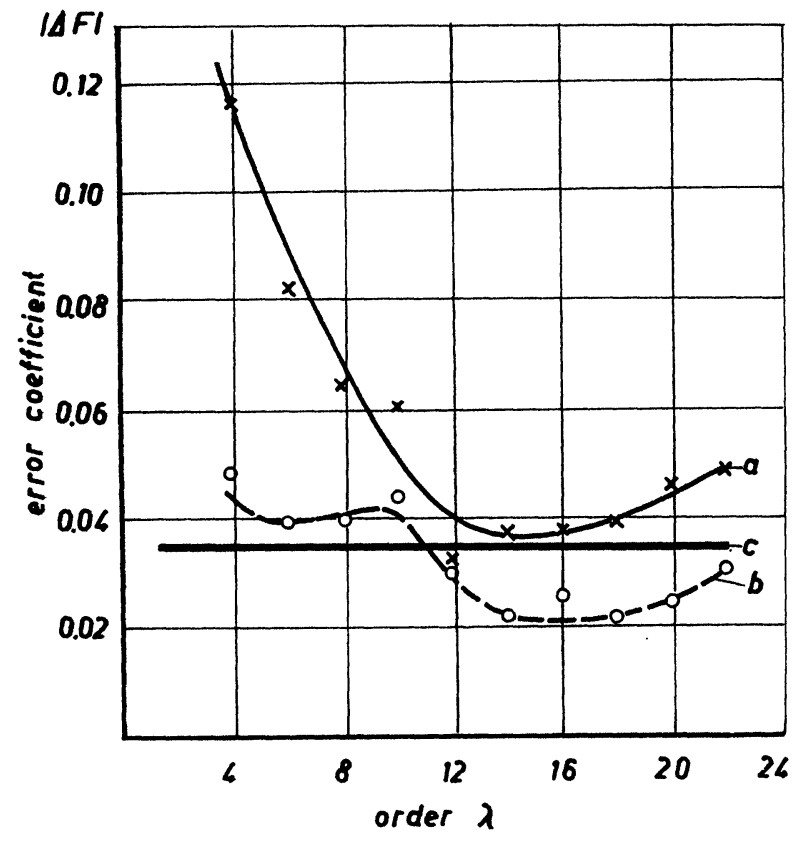

FIGURE 2 Comparison of the error coefficients for a strongly inhomogeneous specimen: (a) sheet sample (b) spherical sample (c) mean value of the statistical errors of several samples.

Then the measurements were repeated with the same specimen, but made into a sphere (curve b). It can be seen that now the lower-order terms are also in the same order of magnitude of the statistical errors which have been estimated from a large number of measurements with different materials (curve c). This shows that the measurements using a spherical sample are no longer influenced by the strong systematical errors due to inhomogeneity although, now as before, the texture is inhomogeneously distributed throughout the sample. The resulting pole density values correpond to the mean value of the texture irrespective of the spatial distribution of the different orientations within the sample.

\section{CONCLUSION}

From the foregoing it must be concluded that a spherical sample is superior to a flat sheet sample in neutron diffraction texture analysis. Furthermore, a sphere of the low quality needed is easy to prepare. Hence the spherical sample method is to be recommended as a standard method in neutron diffraction texture analysis.

\section{REFERENCES}

1. B. N. Brockhouse, Canad. J. Phys. 34, 353 (1953).

2. R. A. Swalin and A. H. Geisler, Trans. AIME 206, 1259 (1956).

3. J. Lanisse, M. Englander and P. Mariell, C. R. Acad. Sci. Paris 249, 2576 (1959).

4. J. Szpunar and J. Dutkiewicz, Bull. Acad. Polonaise Sci. 16, 4, (1968)

5. K. Kleinstück, Wiss. Zeitschrift d. Humboldt Univ.

6. G. E. R. Schulz, in: Realstruktur und Eigenschaften von Reinststoffen (Berlin, 1967).

7. A. Oleś, J. Szpunar and J. Sosnowska, Nukleonika 13, 2, (1968).

8. J. Szpunar, A. Oleś, B. Buras, J. Sosnowska, E. Pietraß, Nukleonika 13, 11-12 (1968)

9. J. Kajamaa, Trans. Met. Soc. AIME 242, 455 (1968).

10. K. Kleinstück and J. Tobisch, Kristall und Technik 3, 344 (1968).

11. H. J. Bunge and J. Tobisch, Z. Metallkunde 53, 471 (1968).

12. H. J. Bunge, J. Tobisch and W. Sonntag, J. Appl. Cryst. 4, 303 (1971).

13. H. J. Bunge and J. Tobisch, J. Appl. Cryst. 5, 27 (1972).

14. U. Schläfer and H. J. Bunge, submitted to Texture.

15. F. Haessner, in: Textures in Research and Practice, (Springer, Berlin, 1969).

16. J. Tobisch, M. Betzl, and P. Reichel, submitted to Bull. Acad. Polonaise Sci.

17. H. J. Bunge, Mathematische Methoden der Texturanalyse (Akademie Verl. Berlin, 1969).

18. L. K. Jetter and B. S. Borie, J. Appl. Phys. 24, 532 (1953).

19. F. Wever and H. Bötticher, Z. Metallkunde 57, 472 (1966).

20. T. Tobisch, to be published.

21. H. J. Bunge, W. T. Roberts, J. Appl. Cryst . 2, 116, (1969). 